Review

\title{
Prognostic Value of Albumin/Globulin Ratio in Survival and Lymph Node Metastasis in Patients with Cancer: A Systematic Review and Meta-analysis
}

\author{
Jieshan Chi1, ${ }^{1,2}$, Qizhi Xie ${ }^{1,2}$, Jingjing Jia ${ }^{1}$, Xiaoma Liu' ${ }^{1}$, Jingjing Sun ${ }^{1}$, Junhui Chen ${ }^{3}$, Li Yi ${ }^{1}$ \\ 1. Department of neurology, Peking University Shenzhen Hospital, Shenzhen 518036, China \\ 2. Shantou University Medical College, Shantou 515041, China \\ 3. Department of Minimally Invasive Intervention, Peking University Shenzhen Hospital, Shenzhen 518036, China \\ $\square$ Corresponding author: Li Yi, email: yilitj@hotmail.com, Tel No.: (+86)13823688918 \\ (c) Ivyspring International Publisher. This is an open access article distributed under the terms of the Creative Commons Attribution (CC BY-NC) license \\ (https://creativecommons.org/licenses/by-nc/4.0/). See http://ivyspring.com/terms for full terms and conditions.
}

Received: 2018.01.11; Accepted: 2018.04.14; Published: 2018.06.12

\begin{abstract}
The impact of albumin to globulin ratio (AGR) on the prognosis of various human cancers has not been well established. Here, a systemic review and meta-analysis has been performed to comprehensively assess the relationships between AGR and lymph node metastasis (LNM) or overall survival (OS). Systematical search through six electronic databases has been carried out to identify reports involving the role of AGR on OS and LNM in human cancers. Hazard ratio $(H R)$, odd ratio $(\mathrm{OR})$ and their $95 \%$ confidence intervals $(95 \% \mathrm{Cl})$ were evaluated through meta-analysis according to standard steps. Of 403 studies retrieved, 14 eligible studies with 4136 patients were included in this study. The analysis based on random-effect model demonstrated that low AGR was significantly associated with poor OS in various cancers $(H R=1.87,95 \% \mathrm{Cl} 1.50-2.34 ; \mathrm{P}<0.001)$. Subsequent results showed a significant increase in the risk of LNM in the low AGR group when compared with high AGR group (HR=2.24; 95\% $\mathrm{Cl}=1.49-3.36 ; \mathrm{P}<0.001$ ). To conclusion, this study suggested that $A G R$ was associated with $O S$ and LNM in cancer patients and AGR may be a potential marker to assess prognosis of cancer patients. However, a large scale of samples and prospective studies are needed in the future to validate the role of $A G R$ in practice.
\end{abstract}

Key words: albumin to globulin ratio, prognosis, cancer, meta-analysis, lymph node metastasis

\section{Introduction}

Cancer is a complex disease that is affected by genetic mutations and various risks in the living environment. Although the mortality of cancer has dropped by about $23 \%$ in the past two decades, cancer is still the biggest problem that plagues human health and the second leading cause of death in the United States. It is estimated that $1,658,210$ new cancer cases occurred in the United States in 2016, and 595,690 cases of them were dead[1]. In recent years, multiple factors were discovered for classifying cancer risks and predicting survival time of cancer patients, such as abnormal expressed genes[2], microRNAs[3] or long non-coding RNAs[4]. However, due to significant individual differences and high costs, these biomarkers cannot be able to applied in clinical decision making process. Hence, it is needed to find an easy-access prognostic factor and stratify patients with different risks in order to make different clinical therapy decisions.

Accumulating evidences revealed that malnutrition and chronically systematic inflammation were responsible for the functional decline of patients with advanced cancer[5]. Serum protein was mainly consisted of two constituents, albumin and globulin. These two kinds of proteins were easy to be measured and closely related to nutritional and inflammatory 
condition of patients. Decreased serum albumin level not only represented malnutrition, but also indicated poor prognosis of ovarian cancer and non-small cell lung cancer patients[6, 7]. As another part of serum protein, globulin in high levels meant the severity of inflammatory response, which resulted in the cardinal features of cancer cachexia[8]. In recent years, increasing studies reported that albumin to globulin ratio (AGR) could be used to predict long-term mortality in various kinds of cancer [9-13]. All these studies indicated that up-regulated AGR was significantly associated with longer survival time in cancer patients and might be an effective prognostic factor for stratifying patients with poor prognosis.

Therefore, this meta-analysis of all published studies was conducted to evaluate the relationship between AGR and clinical outcomes.

\section{Results}

\section{Literature search}

Research through six electronic databases (PubMed, Cochrane, EMBASE, Web of Science, OVID and CNKI) was performed and 403 records of total were identified. Among these studies, 262 of them were duplicate articles and 126 were clearly irrelevant with cancer or studies on animal through the initial screening of the title and abstract. Upon closer inspection on the remaining 36 potential eligible papers, only 14 non-randomized studies pointed out that the relationship between AGR and cancer lymph node metastasis (LNM) or over survival (OS) were deemed to meet all criteria for data extraction. The flowchart of selection process including the justification for final study exclusions was shown in Figure 1.

\section{Characteristics of eligible studies}

All the fourteen eligible studies included in this meta-analysis were published in 2014 to 2017 and eleven different types of cancer were evaluated: two for renal cell carcinomas (RCC), two for colon cancers (CC), two for hepatocellular carcinomas (HCC), one for esophageal squamous cell carcinoma (ESCC), one for upper tract urothelial carcinoma (UTUC), one for natural killer/T-cell lymphoma (NKTCL), one for nasopharyngeal carcinoma (NPC), one for small cell lung cancer (SCLC), one for esophageal cancer (EC), one for glioblastoma (GB), and one for gastric cancer (GC). A total of 4136 patients were chosen and the mean sample size was $\mathrm{N}=329.50$ (range from 105 to 895). All the detective specimens were serum from the patients in their fasting state. The serum albumin and total protein levels were measured spectrophotometrically with an auto-analyzer. AGR was calculated by albumin/ (total protein-albumin). The concrete cut-off value that discriminate low and high AGR. Of these studies, not all studies contained LNM. Across the 14 studies that reported multivariable analyses, only 6 adjusted for cancer stage, 5 adjusted for histologic grade and 3 adjusted for both (Figure 2; Supplementary Fig. 1). Other main characteristics of the eligible studies was presented in the Table 1 . With respect to quality assessment, 100/107 studies were awarded a score of 6 or above, indicating the high quality of the included studies. In addition, the Newcastle-Ottawa Scale (NOS)[14] was examined to evaluate the quality of these studies, and each inclusive publication was regarded as with high quality (Score $>=6$, Supplementary Table S1).

Table 1. Characteristics of studies in this meta-analysis

\begin{tabular}{|c|c|c|c|c|c|c|c|c|c|c|c|c|c|}
\hline \multirow[t]{2}{*}{ Study } & \multirow[t]{2}{*}{ Year } & \multirow[t]{2}{*}{ Country } & \multirow{2}{*}{$\begin{array}{l}\text { Cancer } \\
\text { type }\end{array}$} & \multirow{2}{*}{$\begin{array}{l}\text { Sample } \\
\text { size }\end{array}$} & \multirow[t]{2}{*}{ Cut-off } & \multicolumn{4}{|c|}{ AGR level } & \multirow{2}{*}{$\begin{array}{l}\text { Survival } \\
\text { analysis }\end{array}$} & \multirow{2}{*}{$\begin{array}{l}\text { Multivariate } \\
\text { analysis }\end{array}$} & \multirow{2}{*}{$\begin{array}{l}\text { Hazard ratios }(95 \% \\
\text { CI) }\end{array}$} & \multirow{2}{*}{$\begin{array}{l}\text { Follow-up } \\
\text { (month) }\end{array}$} \\
\hline & & & & & & $\begin{array}{l}\text { Low } \\
\text { level }\end{array}$ & $\begin{array}{l}\text { Low with } \\
\text { LNM }\end{array}$ & $\begin{array}{l}\text { High } \\
\text { level }\end{array}$ & $\begin{array}{l}\text { High with } \\
\text { LNM }\end{array}$ & & & & \\
\hline Zhang J & 2016 & China & $\mathrm{HCC}$ & 105 & 1.18 & 25 & 8 & 80 & 10 & OS & Yes & $5.17(2.72-9.82)$ & 80 (total) \\
\hline Zhang F & 2016 & China & ESCC & 458 & 1.3 & 41 & 22 & 417 & 194 & OS & Yes & $1.318(0.88-1.973)$ & 46.8 (median) \\
\hline Chen Z & 2017 & China & $\mathrm{RCC}$ & 416 & 1.22 & 72 & 5 & 344 & 5 & OS & Yes & $6.529(3.036-14.042)$ & 69.2 (median) \\
\hline Zhang B & 2015 & China & UTUC & 187 & 1.45 & 78 & 3 & 109 & 3 & OS & Yes & $2.12(1.33-3.69)$ & 92 (total) \\
\hline Deng Y & 2016 & China & $\mathrm{HCC}$ & 172 & 1.48 & 105 & $\mathrm{~N} / \mathrm{A}$ & 67 & N/A & OS & Yes & $2.488(1.447-4.292)$ & 120 (total) \\
\hline Bi X & 2016 & China & NKTCL & 331 & 1.3 & 117 & 58 & 214 & 61 & OS & Yes & $1.74(1.18-2.56)$ & 156 (total) \\
\hline Du X & 2014 & China & NPC & 694 & 1.4 & 226 & 172 & 468 & 326 & OS & Yes & $1.439(1.038-1.946)$ & 88 (median) \\
\hline He X & 2017 & China & $\mathrm{RCC}$ & 895 & 1.47 & 371 & 47 & 524 & 19 & OS & Yes & $1.587(1.075-2.326)$ & 69.68 (median) \\
\hline Shibutani M & 2015 & Japan & $\mathrm{CC}$ & 66 & 1.254 & 32 & $\mathrm{~N} / \mathrm{A}$ & 34 & $\mathrm{~N} / \mathrm{A}$ & OS & Yes & $2.247(1.069-4,722)$ & 60 (total) \\
\hline Zhou T & 2016 & China & SCLC & 379 & 1.29 & 91 & $\mathrm{~N} / \mathrm{A}$ & 288 & $\mathrm{~N} / \mathrm{A}$ & OS & Yes & $1.43(1.05-1.95)$ & 15.06 (median) \\
\hline Fujikawa $\mathrm{H}$ & 2017 & Japan & $\mathrm{CC}$ & 248 & 1.32 & $\mathrm{~N} / \mathrm{A}$ & $\mathrm{N} / \mathrm{A}$ & $\mathrm{N} / \mathrm{A}$ & N/A & OS & Yes & $2.67(1.33-5.50)$ & 49.7 (median) \\
\hline Oki S & 2016 & Japan & EC & 112 & 1.41 & $\mathrm{~N} / \mathrm{A}$ & $\mathrm{N} / \mathrm{A}$ & $\mathrm{N} / \mathrm{A}$ & $\mathrm{N} / \mathrm{A}$ & OS & Yes & $0.75(0.31-1.76)$ & 24.9 (median) \\
\hline Xu W & 2017 & China & GB & 166 & 1.75 & 103 & $\mathrm{~N} / \mathrm{A}$ & 63 & N/A & OS & Yes & 1.27 (1.021-2.801) & 14 (median) \\
\hline Toiyama $Y$ & 2016 & Japan & GC & 384 & 1.3793 & $\mathrm{~N} / \mathrm{A}$ & $\mathrm{N} / \mathrm{A}$ & $\mathrm{N} / \mathrm{A}$ & $\mathrm{N} / \mathrm{A}$ & OS & Yes & $1.537(0.890-2.654)$ & 47.6 (median) \\
\hline
\end{tabular}




\section{Overall survival}

All of eligible studies (4136 patients) containing OS were included and classified on the basis of different AGR levels[15-28]. In the light of our research goal, the random effect analysis was applied and the pooled HR was 1.87 (95\% confidence interval (95\% CI) 1.50-2.34, P < 0.001). When the initial meta-analysis was performed, it is found that the data were too heterogeneous $\left(\mathrm{I}^{2}=64.9 \%, \mathrm{P}_{\text {heterogeneity }}<\right.$ 0.001 , Figure $3 \mathrm{~A})$. Due to this reason, firstly the meta-regression analysis was conducted but the source of heterogeneity was still obscure, regardless of year $(\mathrm{P}=0.537)$, country $(\mathrm{P}=0.594)$, cutoff value $(\mathrm{P}=$ $0.058)$, and sample size $(P=0.364$, Table 2$)$. The sensitivity analysis (Figure $3 \mathrm{~B}$ ) was implemented but none of the articles was responsible for heterogeneity. Finally, the heterogeneity was ascribed to two studies, Zhang J (2016)[15] and Chen Z (2017)[16], by the Galbraith Plot (Figure 3C). On the basis of these two studies, meta-analysis was re-run with comparison of other 12 publications. No significant heterogeneity was found $\left(\mathrm{I}^{2}=12.7 \%, \mathrm{P}_{\text {heterogeneity }}=0.32\right)$ and the pooled HR was 1.60 (95\% CI 1.38-1.84, P<0.001, Supplementary Figure S2A) in the random effect analysis. Patients with different AGR levels showed different OS status. This result manifested that the low pretreatment AGR level was associated with poor OS.

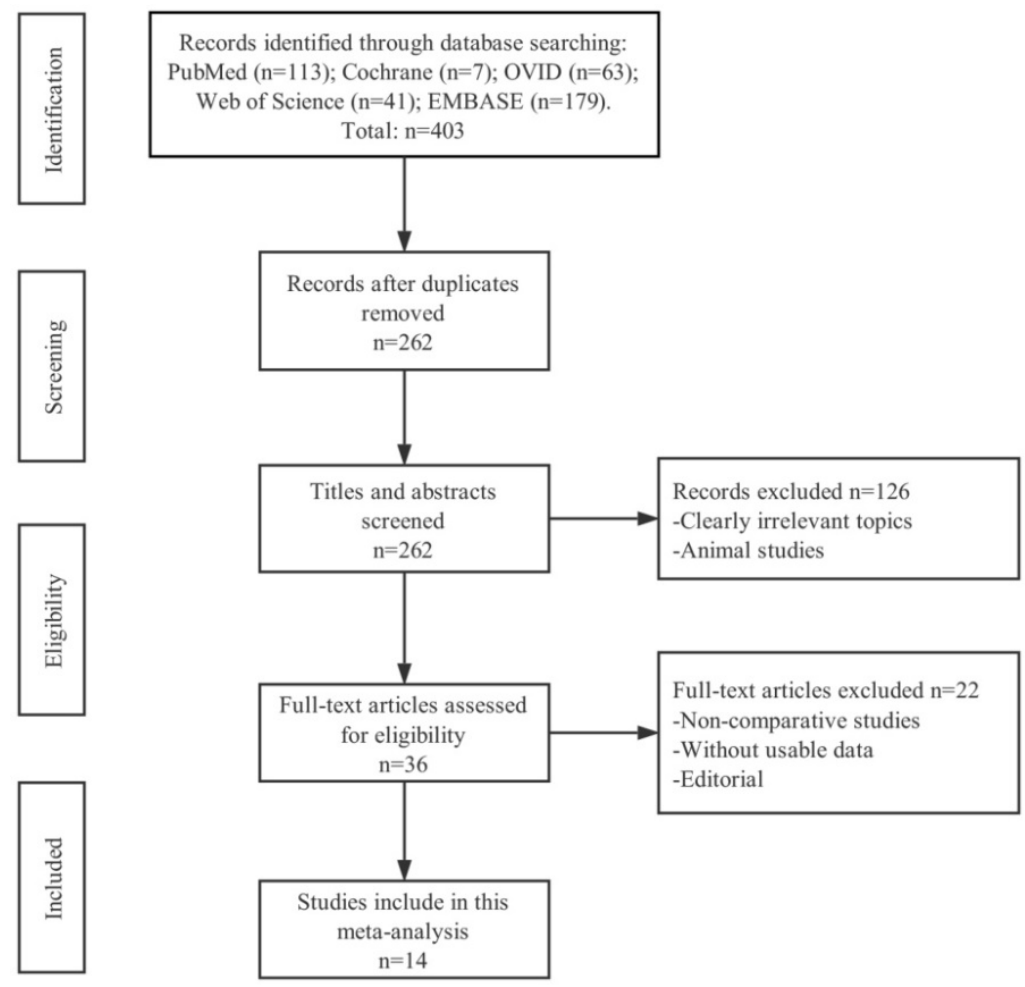

Figure 1. The flow diagram of selecting literature for inclusion.
Table 2. Results of meta-regression on OS

\begin{tabular}{llllll}
\hline & Coefficient & $\begin{array}{l}\text { Standard } \\
\text { error }\end{array}$ & t & p value & 95\%CI \\
\hline Year & 0.0929285 & 1505306 & 0.62 & 0.537 & $-0.202106,0.387963$ \\
Country & 0.1671462 & 0.3139068 & 0.53 & 0.594 & $-0.4480988,0.7823921$ \\
Cut-off & -0.1631723 & 0.59902 & -1.9 & 0.058 & $-3.317096,0.0536508$ \\
Sample size & -0.0004788 & 0.0005273 & -0.9 & 0.364 & $-0.0015123,0.0005546$ \\
& & & 1 & & \\
\hline
\end{tabular}

Table 3. Results of this meta-analysis.

\begin{tabular}{lllllll}
\hline Outcome & $\begin{array}{l}\text { No.of } \\
\text { studies }\end{array}$ & $\begin{array}{l}\text { No.of } \\
\text { patients }\end{array}$ & HR/OR(95\%CI) & P & \multicolumn{2}{c}{ Heterogeneity } \\
\cline { 6 - 7 } & 14 & 4136 & $1.87(1.50-2.34)$ & $<0.001$ & 64.9 & $<0.001$ \\
\hline OS & 7 & 3086 & $2.24(1.49-3.36)$ & $<0.001$ & 59.2 & 0.023 \\
LNM & 7 & 2978 & $3.16(1.99-4.99)$ & $<0.001$ & 72.7 & 0.003 \\
TS & 6 &
\end{tabular}

OS: overall survival, LNM: lymph node metastasis, TS: tumor stage, HR: hazard ratios, OR: odds ratios, No: number, CI: confidence interval.

\section{Lymph node metastasis and tumor stage}

Only seven studies (3086 patients) containing LNM were included based on different AGR levels. The random effect analysis was conducted and the pooled OR of these seven articles was 2.24 (95\% CI 1.50-3.36, $\mathrm{P}<0.001$; Figure 4A). And significant heterogeneity was detected $\left(\mathrm{I}^{2}=59.2 \%\right.$, $\mathrm{P}_{\text {heterogeneity }}=$ 0.023 , Figure $4 \mathrm{~A})$. After post hoc sensitivity analysis, there is no manifestation of the chief cause of significant heterogeneity (Figure 4B). Besides, the meta-regression and subgroup analyses were not allowed to perform effectively because there were only seven articles in this analysis. The result indicated that cancer patients with low AGR level were more prone to develop lymph node metastasis. Six of seven studies (2978 patients) with LNM containing tumor stage were included based on different AGR levels. The pooled OR was 3.16 (95\% CI 1.99-4.99, P<0.001, Supplementary Figure S2B). However, it was shown that significant heterogeneity also existed in this result $\left(\mathrm{I}^{2}=72.7 \%\right.$, $P_{\text {heterogeneity }}=0.003$, Supplementary Figure S2B). After sensitivity analysis, it can be found that no publication affected the result (Supplementary Figure S2C). And as mentioned above, due to the number of the articles, the meta-regression and subgroup analyses were not allowed to perform effectively. Although there was much significant expression of heterogeneity, the conclusion still can be reached that the low AGR had a certain correlation with poor tumor stage OR.

All of the results of this metaanalysis were shown in Table 3. 


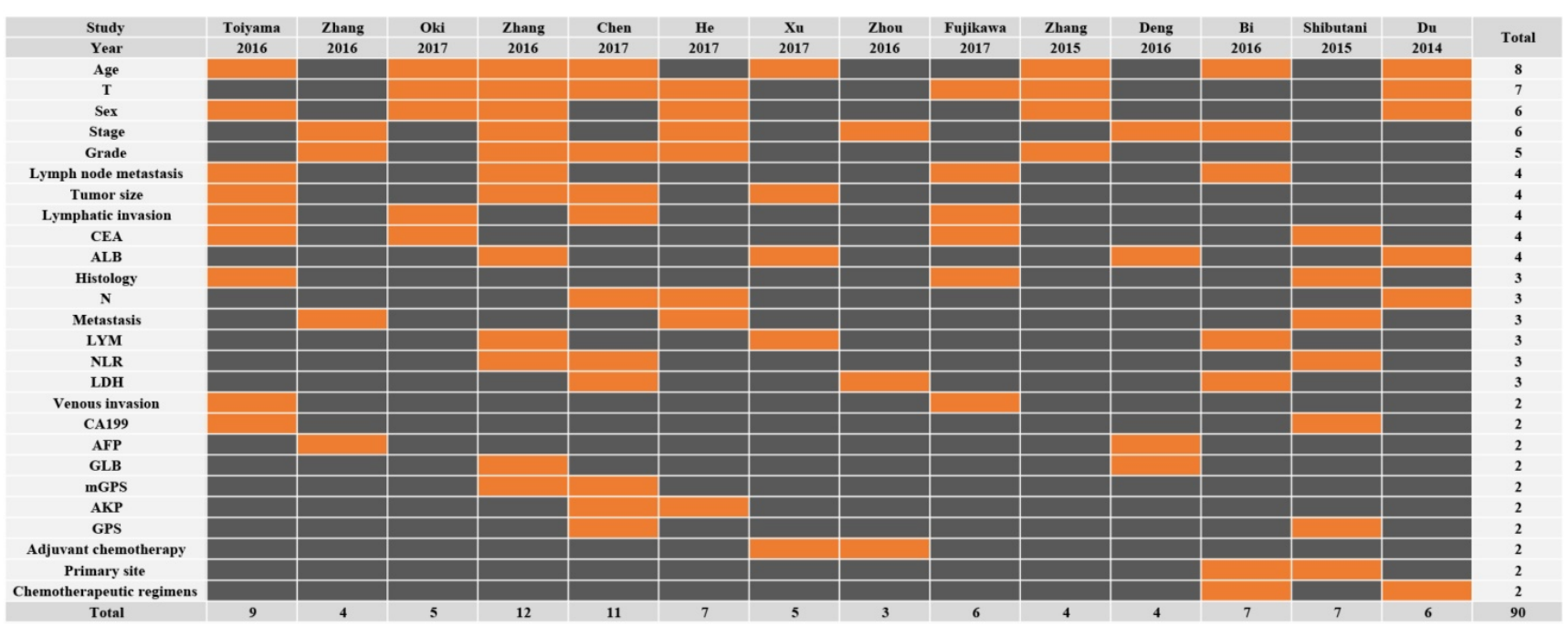

Figure 2. The covariates used in the multivariable models of each study. This is a representative data microarray illustrating all of the covariates studied (X-axis: Studies; Y-axis: Covariates). Only the factors used two or more times are shown in this figure for convenience; refer to Supplementary Figure S1 for a data microarray illustrating all covariates studied. Rows are in descending order based on how many times each covariate was included in a multivariable model. Orange $=$ included in the multivariable models. Black = not included in the multivariable models. T, T stage; CEA, Carcinoembryonic antigen; ALB, Albumin; N, N stage; LYM, Lymphocyte; NLR, Neutrophil to lymphocyte ratio; LDH, Lactate dehydrogenase; CA199, Carbohydrate antigen 199; AFP, Alpha fetoprotein; GLB, Globulin; AKP, Alkaline phosphatase; GPS, Glasgow Prognostic Score.

A.

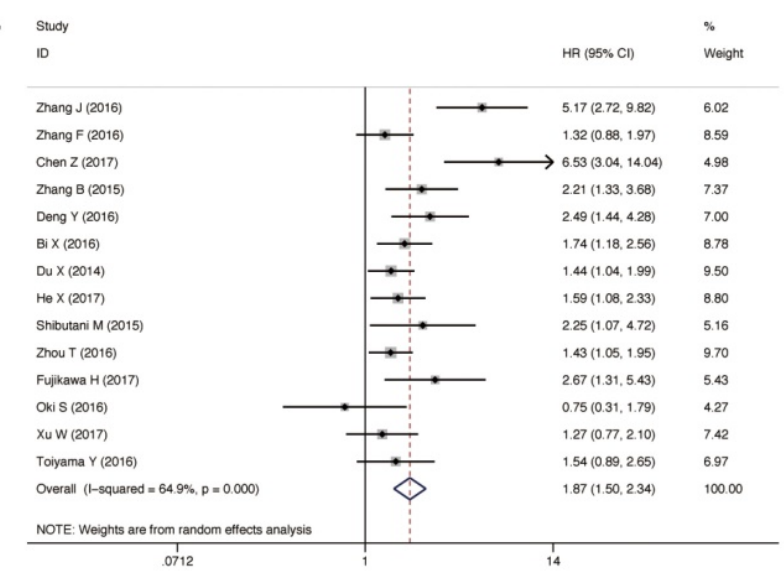

B.

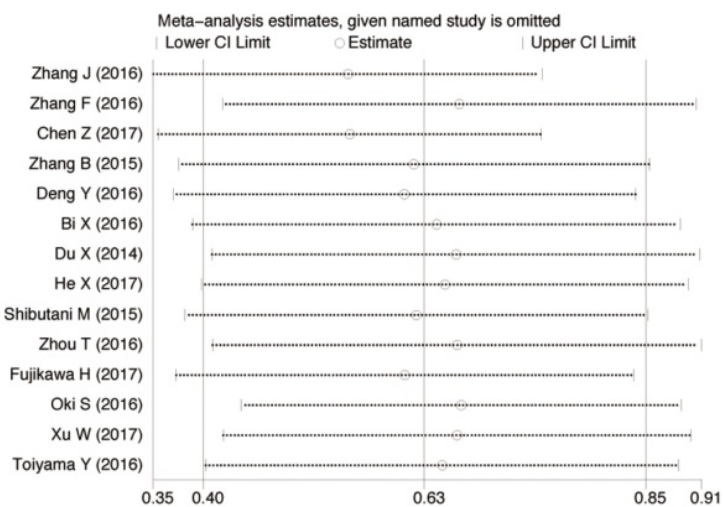

C. $\mathrm{b} / \mathrm{se}$

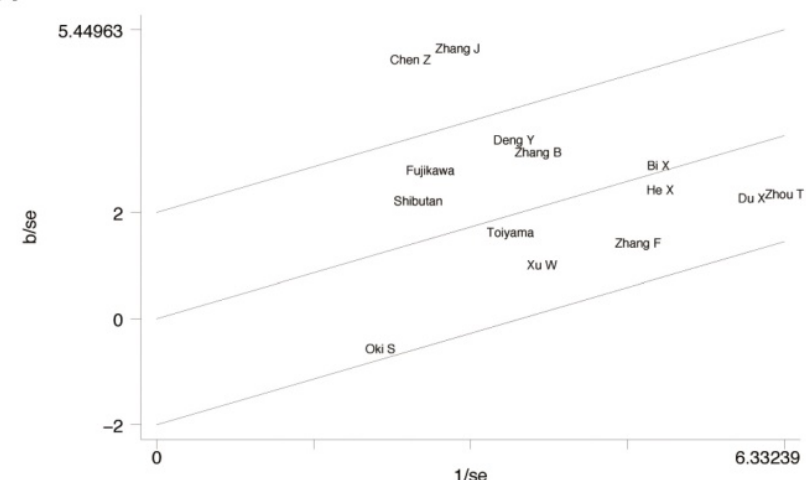

Figure 3. Association between AGR and OS. A) The Forest Plot of HR with 14 eligible publications for the correlation between AGR and OS. B) Sensitivity analysis of the correlation between AGR and OS with 14 eligible publications. C) Galbraith Plot of heterogeneity analysis with 14 eligible publications.

\section{Publication bias}

Publication bias was assessed by funnel plot which was conducted by Begg's tests. The result suggested that no asymmetric was existed. Egger's test was also performed to confirm the data. There was no significant publication bias observed in both 
A.

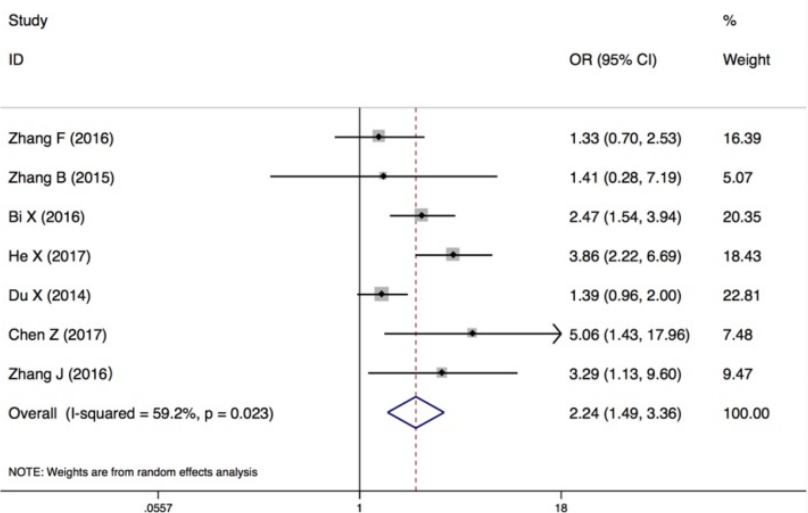

B.

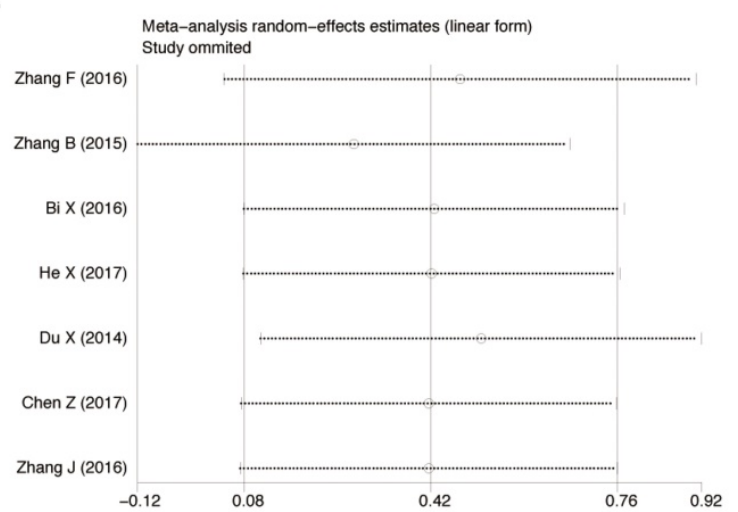

Figure 4. Association between AGR and LNM. A) Forest Plot of OR for the correlation between AGR and LNM. B) Sensitivity analysis of the correlation between AGR and LNM.
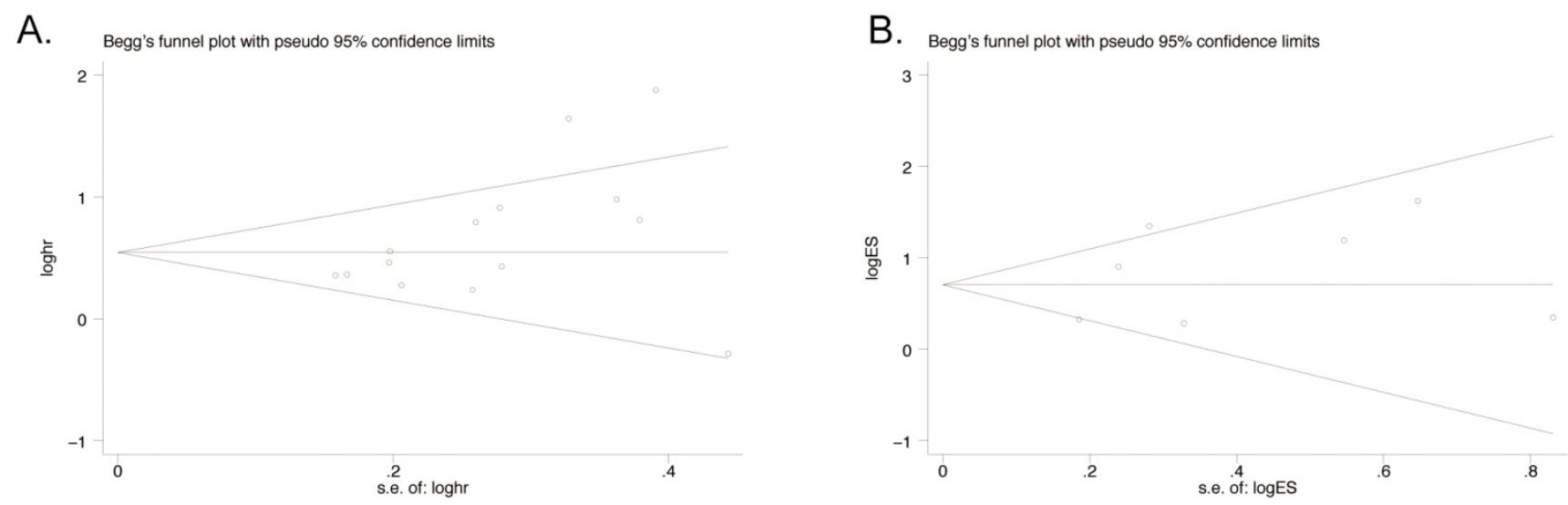

Figure 5. Funnel Plot analyses of publication bias in OS and LNM (Egger's test): A) OS group; B) LNM group.

OS group with 14 studies (Egger's test, $\mathrm{P}=0.08$; Begg's test, $\mathrm{P}=0.062$, Figure $5 \mathrm{~A}$ ) and LNM group with 7 studies (Egger's test, $\mathrm{P}=0.400$; Begg's test, $\mathrm{P}=0.764$, Figure 5B).

\section{Discussion}

Albumin and globulin were the major components of human total serum proteins. Previous studies indicated that low serum albumin was a negative predictor of overall survival rate in several tumors[29] including colorectal[30-32], gastric[33], pancreatic[34, 35], hepatocellular, breast[29, 36], lung[29], and ovarian[7, 29]. Low serum albumin would lead to hypoproteinemia or malnutrition and further effect the survival duration and life quality of patients. Malnutrition would weaken the cellular and humoral immunity, phagocytic function and other defense mechanisms of patients with cancer. It was particularly noteworthy that though serum albumin was at normal levels or slightly low, it led to poor prognosis in cancer patients because of its insufficiency of treatment[13]. Considering patients' nutritional conditions, several studies pointed out that serum albumin was involved in the inflammatory process. During the process of cancer formation or development, the role of inflammatory microenvironment has been mainly emphasized[37, 38]. If there was a sufficient amount of serum albumin, polyunsaturated fatty acids (especially arachidonic acid, eicosapentaenoic acid and docosahexaenoic acid) will be mobilized from the liver, which was helpful to form cell biologically active lipids such as lipoxins, resolvins and protective[39].

The relationship between decreased serum albumin and chronic infections would be a good explanation for wide link to carcinogenesis. The globulin mainly functioned as an important role in immunity and inflammation, so the elevated serum globulin was associated with several kinds of chronic inflammatory diseases. Increase in inflammatory cytokines in serum globulin would contribute to the elevated serum proteins and the suppression of serum albumin biosynthesis[40-43]. Furthermore, it was indeed proved provoking that elevated calculated globulins would lead to pool outcomes in cancer patients with normal serum albumin[10]. Thus, there was sufficient biological plausibility to combine the albumin and globulin to assess the outcomes of 
patients with cancer instead of single albumin or globulin due to the interaction of two variables, nutrition and inflammation. Moreover, AGR was a promising predictor because of its easy access and little cost in clinical practice. Therefore, it's assumed that the more effective and comprehensive AGR may be a potential biomarker for the prognosis of cancer patients.

To the best of our knowledge, this meta-analysis was the first to assess the correlation of AGR with the overall survival and lymph node metastasis of patients with various cancers. In this study, the outcomes of 4136 patients from 14 available studies were integrated indicating that a low AGR was significantly associated with poor OS HR $(1.87,95 \%$ CI 1.50-2.34, $\mathrm{P}<0.001)$ in various cancers, in spite of the exist of heterogeneity. After removal of two of these publications that were contributed to heterogeneity, the relationship between AGR and OS was still statistically significant (HR 1.586, 95\% CI 1.392-1.806, $\mathrm{P}<0.001)$. The analyses between AGR and lymph node metastasis were performed, indicating that a low AGR was a negative maker for LNM. When patients were stratified by tumor stages, a trend can be found to represent the relationship between decreased AGR level and higher tumor stage. In addition, the researches, stratified by AGR tertile, further revealed that with the increase of AGR, the overall survival of cancer patients boosted $[9,10,44]$.

In the meta-analysis, there were some limitations need to be improved. The first one was the limited number of included publications, especially the studies about the relationship between AGR and LNM, and the results about pooled HR of overall survival and pooled OR of LNM may not credible enough. It is possible that relevant studies may have been missed, as our systematic review was based on only 6 databases (PubMed, Cochrane, EMBASE, Web of Science, OVID and CNKI). Theoretically, we could have searched for possible missing articles in grey literature, relevant books, databases such as Google Scholar and other potential resources. In that way, our search strategy would be more sufficient, and the risk of publication bias would be reduced. The second one was significant heterogeneity among the included publications. To address this problem, the meta-regression analysis on the basis of Galbraith Plot and sensitivity analyses were performed, and finally the principal responsibility articles were excluded in order to control heterogeneity. But heterogeneity could not be completely eliminated and the reason why the principal responsibility articles were so heterogeneous did not be explained reasonably. This may have been the result of the combination of multiple studies that are known to be heterogeneous in terms of cancer site, provenance of patient populations, cut-off types and a small sample size due to the limited number of included studies for each cancer type. Third, all the eligible publications were retrospective due to the absence of prospective studies. Forth, only about $20 \%$ of the reported prognostic effects were adjusted for the two classically most approved predictors of cancer prognosis. One vital aspect in ascribing availability to a novel biomarker is their capability to add further predictive value which even over the already possible using known prognostic factors. Therefore, the further investigations were required to confirm whether the AGR is a potential prognostic predictor in cancer patients.

In conclusion, this meta-analysis demonstrated that low AGR was significantly associated with shorter OS and higher lymph node metastasis rate in cancer patients. The further prospective studies were required to figure out the critical role of AGR for outcome in patients with cancer.

\section{Materials and Methods}

\section{Search strategy}

The present study was performed according to the Preferred Reporting Items for Systematic Reviews and Meta-Analyses (PRISMA) guideline. And literature search was executed on PubMed, Cochrane, EMBASE, Web of Science, OVID and Chinese National Knowledge Infrastructure (CNKI). No language or data restrictions were imposed and the searching was update to Apr. 28, 2017. The keywords of search included "albumin globulin ratio" or "Albumin-globulin ratio" or "AGR" and "carcinoma" or "cancer" or "tumor" or "neoplasm".

\section{Inclusion and exclusion criteria}

The studies concerning the relationship between AGR level and prognosis of cancer were collected by two of the authors independently. Articles were checked with abstract and titles initially and needed further screening with following criteria. Inclusion criteria were as follows: (1) investigation of the association between AGR level and prognosis of cancer; (2) cancer patients were put into two groups (high/low) according to the level of AGR which was calculated as AGR = albumin/ (total proteinalbumin); (3) available clinical parameters included HR, OS and/or LNM; (4) cancer diagnosis was confirmed by pathological examination. The exclusion criteria were as follows: (1) duplicate or irrelevant or nonhuman research; (2) lack of useful data; (3) the following type of studies: letters, reports, reviews, expert opinions and editorials; (4) lack of available grouping. 


\section{Data extraction}

All the eligible studies data were reviewed and extracted independently by two of the authors. Each disagreement was discussed sufficiently until the authors reached a consensus to make sure the veracity of all the information. The following data of each studies were extracted: first author, year of publication, country of origin; total number of participants, cancer type, follow-up, cutoff value, number of patients with AGR in different group, HRs of AGR for OS and the $95 \% \mathrm{CI}$ and their $\mathrm{P}$ value, number of patients with LNM and/or tumor stage in different group.

\section{Statistic method}

The pooled HR were calculated with the extracted HRs and its 95\% CIs from the original articles. The heterogeneity of combined HRs or ORs was preliminarily evaluated by Forest Plots. Then the exact statistical heterogeneity assessment was performed by Cochran's Q test and Higgins I-squared statistic, which measured the percentage of the total variation across studies[45]. $\mathrm{P}<0.1$ or $\mathrm{I}^{2}>50 \%$ was considered as the existence of significant heterogeneity. In view of our research goal, the random-effect model was applied[46]. Metaregression, Galbraith Plot and sensitivity analysis were performed to detect the origin of heterogeneity when the data was too heterogeneous. The publication bias was detected by both of the Begg's and Egger's tests. In the meta-analysis, STATA version 14.0 was used to carry out the statistical analyses, and $\mathrm{P}<0.05$ was considered as statistically significant.

\section{Supplementary Material}

Supplementary figures and table. http://www.jcancer.org/v09p2341s1.pdf

\section{Competing Interests}

The authors have declared that no competing interest exists.

\section{References}

1. Siegel RL, Miller KD, Jemal A. Cancer statistics, 2016. CA: a cancer journal for clinicians. 2016; 66: 7-30.

2. Collier LS, Largaespada DA. Transforming science: cancer gene identification. Current opinion in genetics \& development. 2006; 16: 23-9.

3. He Y, Lin J, Kong D, Huang M, Xu C, Kim TK, et al. Current State of Circulating MicroRNAs as Cancer Biomarkers. Clin Chem. 2015; 61: 1138-55.

4. Cheng W, Zhang Z, Wang J. Long noncoding RNAs: new players in prostate cancer. Cancer letters. 2013; 339: 8-14.

5. McMillan DC, Watson WS, O'Gorman P, Preston T, Scott HR, McArdle CS. Albumin concentrations are primarily determined by the body cell mass and the systemic inflammatory response in cancer patients with weight loss. Nutrition and cancer. 2001; 39: 210-3.

6. Espinosa E, Feliu J, Zamora P, Gonzalez Baron M, Sanchez JJ, Ordon ez A, et al. Serum albumin and other prognostic factors related to response and survival in patients with advanced non-small cell lung cancer. Lung cancer (Amsterdam, Netherlands). 1995; 12: 67-76.
7. Asher V, Lee J, Bali A. Preoperative serum albumin is an independent prognostic predictor of survival in ovarian cancer. Medical oncology (Northwood, London, England). 2012; 29: 2005-9.

8. McMillan DC. Systemic inflammation, nutritional status and survival in patients with cancer. Current opinion in clinical nutrition and metabolic care. 2009; 12: 223-6.

9. Azab BN, Bhatt VR, Vonfrolio S, Bachir R, Rubinshteyn V, Alkaied H, et al. Value of the pretreatment albumin to globulin ratio in predicting long-term mortality in breast cancer patients. American journal of surgery. 2013; 206: 764-70.

10. Azab B, Kedia S, Shah N, Vonfrolio S, Lu W, Naboush A, et al. The value of the pretreatment albumin/globulin ratio in predicting the long-term survival in colorectal cancer. International journal of colorectal disease. 2013; 28: 1629-36.

11. Zhang B, Yu W, Zhou LQ, He ZS, Shen C, He Q, et al. Prognostic Significance of Preoperative Albumin-Globulin Ratio in Patients with Upper Tract Urothelial Carcinoma. PloS one. 2015; 10: e0144961.

12. Deng $Y$, Pang $Q$, Miao RC, Chen W, Zhou YY, Bi JB, et al. Prognostic significance of pretreatment albumin/globulin ratio in patients with hepatocellular carcinoma. OncoTargets and therapy. 2016; 9: 5317-28.

13. Liu J, Dai Y, Zhou F, Long Z, Li Y, Liu B, et al. The prognostic role of preoperative serum albumin/globulin ratio in patients with bladder urothelial carcinoma undergoing radical cystectomy. Urologic oncology. 2016; 34: 484.e1-.e8.

14. Stang A. Critical evaluation of the Newcastle-Ottawa scale for the assessment of the quality of nonrandomized studies in meta-analyses. European journal of epidemiology. 2010; 25: 603-5.

15. Zhang J, Liu X, Yang Z, Chen Y, Luo R. The pretreatment albumin to globulin ratio, a validated biomarker, predicts prognosis in hepatocellular carcinoma. Journal of BUON : official journal of the Balkan Union of Oncology. 2016; 21: 925-34.

16. Chen Z, Shao Y, Yao H, Zhuang Q, Wang K, Xing Z, et al. Preoperative albumin to globulin ratio predicts survival in clear cell renal cell carcinoma patients. Oncotarget. 2017.

17. Zhang $F$, Sun $P$, Wang ZQ Wang de $S$, Wang $Y$, Zhang DS, et al. Low preoperative albumin-globulin score predicts favorable survival in esophageal squamous cell carcinoma. Oncotarget. 2016; 7: 30550-60.

18. Zhang B, Yu W, Zhou LQ, He ZS, Shen C, He Q, et al. Prognostic significance of preoperative albumin-globulin ratio in patients with upper tract urothelial carcinoma. PLoS ONE. 2015; 10 .

19. Deng $Y$, Pang Q, Miao RC, Chen W, Zhou YY, Bi JB, et al. Prognostic significance of pretreatment albumin/ globulin ratio in patients with hepatocellular carcinoma. OncoTargets and Therapy. 2016; 9: 5317-28.

20. Bi XW, Wang L, Zhang WW, Yan SM, Sun P, Xia Y, et al. The pretreatment albumin to globulin ratio predicts survival in patients with natural killer/T-cell lymphoma. PeerJ. 2016; 4: e1742.

21. Du XJ, Tang LL, Mao YP, Sun Y, Zeng MS, Kang TB, et al. The pretreatment albumin to globulin ratio has predictive value for long-term mortality in nasopharyngeal carcinoma. PLoS ONE. 2014; 9.

22. He X, Guo S, Chen D, Yang G, Chen X, Zhang Y, et al. Preoperative Albumin to Globulin Ratio (AGR) as Prognostic Factor in Renal Cell Carcinoma. Journal of Cancer. 2017; 8: 258-65.

23. Shibutani M, Maeda $K$, Nagahara H, Ohtani H, Iseki $Y$, Ikeya $T$, et al. The pretreatment albumin to globulin ratio predicts chemotherapeutic outcomes in patients with unresectable metastatic colorectal cancer. BMC Cancer. 2015; 15.

24. Zhou $\mathrm{T}, \mathrm{He} X$, Fang $\mathrm{W}$, Zhan J, Hong S, Qin T, et al. Pretreatment albumin/globulin ratio predicts the prognosis for small-cell lung cancer. Medicine (United States). 2016; 95.

25. Fujikawa $H$, Toiyama $Y$, Inoue $Y$, Imaoka $H$, Shimura $T$, Okigami $M$, et al. Prognostic Impact of Preoperative Albumin-to-Globulin Ratio in Patients with Colon Cancer Undergoing Surgery with Curative Intent. Anticancer research. 2017; 37: 1335-42.

26. Oki S, Toiyama $\mathrm{Y}$, Okugawa $\mathrm{Y}$, Shimura $\mathrm{T}$, Okigami $\mathrm{M}$, Yasuda $\mathrm{H}$, et al. Clinical burden of preoperative albumin-globulin ratio in esophageal cancer patients. Am J Surg. 2017.

27. Xu WZ, Li F, Xu ZK, Chen X, Sun B, Cao JW, et al. Preoperative albumin-to-globulin ratio and prognostic nutrition index predict prognosis for glioblastoma. OncoTargets and Therapy. 2017; 10: 725-33.

28. Toiyama Y, Yasuda H, Ohi M, Yoshiyama S, Araki T, Tanaka K, et al. Clinical impact of preoperative albumin to globulin ratio in gastric cancer patients with curative intent. American Journal of Surgery. 2017; 213: 120-6.

29. Gupta D, Lis CG. Pretreatment serum albumin as a predictor of cancer survival: a systematic review of the epidemiological literature. Nutrition journal. 2010; 9: 69 .

30. Lai CC, You JF, Yeh CY, Chen JS, Tang R, Wang JY, et al. Low preoperative serum albumin in colon cancer: a risk factor for poor outcome. Int J Colorectal Dis. 2011; 26: 473-81.

31. Boonpipattanapong T, Chewatanakornkul S. Preoperative carcinoembryonic antigen and albumin in predicting survival in patients with colon and rectal carcinomas. Journal of clinical gastroenterology. 2006; 40: 592-5.

32. Sun LC, Chu KS, Cheng SC, Lu CY, Kuo CH, Hsieh JS, et al. Preoperative serum carcinoembryonic antigen, albumin and age are supplementary to UICC staging systems in predicting survival for colorectal cancer patients undergoing surgical treatment. BMC Cancer. 2009; 9: 288

33. Onate-Ocana LF, Aiello-Crocifoglio V, Gallardo-Rincon D, Herrera-Goepfert $\mathrm{R}$, Brom-Valladares $\mathrm{R}$, Carrillo JF, et al. Serum albumin as a significant 
prognostic factor for patients with gastric carcinoma. Ann Surg Oncol. 2007; 14: $381-9$.

34. Siddiqui A, Heinzerling J, Livingston EH, Huerta S. Predictors of early mortality in veteran patients with pancreatic cancer. Am J Surg. 2007; 194: 362-6.

35. Ruiz-Tovar J, Martin-Perez E, Fernandez-Contreras ME, Reguero-Callejas ME, Gamallo-Amat C. Impact of preoperative levels of hemoglobin and albumin on the survival of pancreatic carcinoma. Revista espanola de enfermedades digestivas : organo oficial de la Sociedad Espanola de Patologia Digestiva. 2010; 102: 631-6.

36. Lis CG, Grutsch JF, Vashi PG, Lammersfeld CA. Is serum albumin an independent predictor of survival in patients with breast cancer? JPEN Journal of parenteral and enteral nutrition. 2003; 27: 10-5.

37. Candido J, Hagemann T. Cancer-related inflammation. Journal of clinical immunology. 2013; 33 Suppl 1: S79-84.

38. Colotta F, Allavena P, Sica A, Garlanda C, Mantovani A. Cancer-related inflammation, the seventh hallmark of cancer: links to genetic instability. Carcinogenesis. 2009; 30: 1073-81.

39. Das UN. Albumin to globulin ratio and/or plasma albumin in predicting long-term mortality. Am J Surg. 2014; 208: 157-8.

40. O'Connell TX, Horita TJ, Kasravi B. Understanding and interpreting serum protein electrophoresis. American family physician. 2005; 71: 105-12.

41. Vavricka SR, Burri E, Beglinger C, Degen L, Manz M. Serum protein electrophoresis: an underused but very useful test. Digestion. 2009; 79: 203-10.

42. Kaysen GA, Dubin JA, Muller HG, Mitch WE, Rosales LM, Levin NW. Relationships among inflammation nutrition and physiologic mechanisms establishing albumin levels in hemodialysis patients. Kidney international. 2002; 61: 2240-9.

43. Gabay C, Kushner I. Acute-phase proteins and other systemic responses to inflammation. The New England journal of medicine. 1999; 340: 448-54.

44. Duran $\mathrm{AO}$, Inanc $\mathrm{M}$, Karaca $\mathrm{H}$, Dogan I, Berk $\mathrm{V}$, Bozkurt $\mathrm{O}$, et al. Albumin-globulin ratio for prediction of long-term mortality in lung adenocarcinoma patients. Asian Pacific journal of cancer prevention : APJCP. 2014; 15: 6449-53.

45. Higgins JP, Thompson SG, Deeks JJ, Altman DG. Measuring inconsistency in meta-analyses. BMJ (Clinical research ed). 2003; 327: 557-60.

46. Higgins JP. Commentary: Heterogeneity in meta-analysis should be expected and appropriately quantified. Int J Epidemiol. 2008; 37: 1158-60. 